\title{
GOVERNANÇA DA ÁGUA NO BRASIL: UMA CONTRIBUIÇÃO BIBLIOMÉTRICA
}

\author{
D. VASCONCELOS*, N. GONDIM, P. A. HORDONES, A. C. SILVA e M. R. BARROS \\ Universidade Federal de Goiás - UFG \\ debora.pvasconcelos@gmail.com*
}

Artigo submetido em 09/07/2016 e aceito em 31/10/2016

DOI: $10.15628 /$ holos.2016.4814

\section{RESUMO}

A água é indispensável para o desenvolvimento social, pessoal e econômico, sendo assim é necessário adotar novas práticas de relação entre governo, sociedade e o uso sustentável da mesma. Desde 2012, vários municípios brasileiros têm se deparado com reduções no volume pluviométrico, delineando um cenário de crise hídrica. Diante disso, esse trabalho através de um estudo bibliométrico com 137 artigos compreende a dimensão e as características da governança da água no contexto nacional. Destacou-se um total de 1120 citações desses artigos, o que dá uma média por ano de
65,88 citações e 8,18 por artigo, além disso a amostra apresentou 1,82 autores por artigo, o autor com maior número de publicações foi Jacobi, P. R. e o periódico com maior destaque em volume de publicações foi o Ambiente \& Sociedade. Dessa forma, nota-se que o tema não é pouco discutido e a transição da inovadora governança da água, trata-se da reintegração da natureza na história, pois, a dimensão cultural aponta que são necessárias mudanças nos nossos valores, instituições e modo de vida na medida em que não se pode viver bem se a natureza é destruída.

PALAVRAS-CHAVE: Crise Hídrica, Revisão da Literatura, Gestão.

\section{WATER GOVERNANCE IN BRAZIL: A BIBLIOMETRIC CONTRIBUTION}

\begin{abstract}
Water is essential for the social, personal and economic, so it is necessary to adopt new practices of relationship between government, society and the sustainable use of it. Since 2012, several municipalities have faced reductions in rainfall volume, outlining a water crisis scenario. Therefore, this work through a bibliometric study of 137 articles comprises the water governance features in the national context. stood out a total of 1120 citations of these articles, which gives an average per year of 65.88 and 8.18 citations per article, besides
\end{abstract}

the sample showed 1.82 authors per article, the author with the highest number of publications was Jacobi, P. R. and the periodic most notably in volume of publications is Environment \& Society. Thus, it is noted that the issue is not little discussed and the transition of innovative water governance, it is the reintegration of nature in history, therefore, the cultural dimension shows that changes are needed in our values, institutions and way of life to the extent that it cannot live well if the nature is

KEYWORDS: Hydro crisis, Literature Review, Management. 


\section{INTRODUÇÃO}

A política da água teve início no País com as novas relações entre governo e sociedade, fundadas pela Constituição Federal de 1988, com os princípios de descentralização do poder e participação popular. A partir disso, para Costa e Mertens (2015), a gestão dos recursos hídricos brasileiros não é mais uma questão de governo, mas sim de governança, pois, desde 2012, o Brasil encontra-se com reduções de chuvas, apresentando um cenário de escassez hídrica (Jacobi, Cibim \& Leão, 2015). O conceito de governança é o ato de governar ou governação, isto é, uma administração ativa. Sabendo disso, a governança da água é vista como uma inovação. Além de defender um novo paradigma na gestão dos recursos hídricos, há propostas de transformações na infraestrutura política.

Normalmente encontram-se estudos sobre a crise hídrica brasileira voltados mais para a cidade de São Paulo, a qual é o maior município da Região Metropolitana de São Paulo, com mais de onze milhões de habitantes (Jacobi, Fracalanza \& Silva-Sánchez, 2015). Em contrapartida, as pesquisas internacionais são mais voltadas aos pressupostos políticos respectivos à democracia no contexto geral (Martins, 2015). Contudo, esse estudo caracteriza-se pela revisão bibliográfica sobre a inovação da governança hídrica relacionada a escassez da água em âmbito nacional.

Em uma perspectiva sociológica, Souza, Silva e Barbosa (2014), defendem que a definição de problemas é subjetiva, dependente dos sistemas de significado e comportamento dos indivíduos. Entretanto, independente dessas definições a água é uma herança natural estratégica.

Com isso, a pesquisa almeja trazer maiores evidências das características dos estudos sobre a crise hídrica brasileira. Associou-se à interpretação do conceito de governança da água como práticas de planejamento, implementação e gestão de políticas de recursos hídricos com as políticas sustentáveis que se fundamentam em aspectos econômicos, ambientais e sociais, que são base para a elaboração das políticas ambientais, além do fenômeno climático e o uso desnecessário da água.

Tundisi (2008), assegura que a situação atual brasileira referente a água apresenta dimensões local, regional, continental e planetária. Sabendo que, o modelo de gestão das águas no Brasil, em vigor atualmente, se iniciou nos anos 1970 para os 1980 (Campos \& Fracalanza, 2010), pode se definir que a situação hídrica brasileira é um conjunto do gerenciamento do recurso com a escassez pluvial ou um conjunto de problemas ambientais com problemas relacionados à economia e ao desenvolvimento social.

Com isso, os desafios que se colocam a gestão da água tornaram-se cada vez mais global desde a década de 1970. Nesse contexto, a problemática que se coloca, é compreender a dimensão e as características dos estudos sobre governança da água no contexto nacional, a evolução das publicações durante os anos, quem são os principais autores, quais os principais periódicos nos quais os trabalhos foram publicados e o comportamento do tema no meio cientifico, como é sua visibilidade e citação. 


\section{REVISÃO BIBLIOGRÁFICA}

\subsection{Realidade Brasileira dos Recursos Hídricos}

Somente na cidade de São Paulo, a estrutura hidrográfica é formada por 103 bacias contidas em seu território (Jacobi, Fracalanza \& Silva-Sanchéz, 2015). Porém, em âmbito nacional, a atenção é voltada para o recurso água e a reforma das instituições que a gerem, desde meados da década de 1980, conforme Martins (2015).

A água é um recurso natural renovável, mas pode se esgotar, caso haja mal-uso. Nessa perspectiva, é fundamental a maneira pela qual as instituições entendem a situação e a estrutura correspondente e definem os critérios para tomada de decisões (Souza, Silva \& Barbosa, 2014), pois a água é indispensável para o desenvolvimento econômico, social e pessoal. De acordo com Wolkmer e Pimmel (2013), sem água a vida não existe.

No estudo de Fracalanza, Jacob e Eça (2013), fez-se uma distinção entre os valores comerciais da água e os valores de uso. Tiveram como resultado que, a água enquanto substância necessária à vida possibilita a discussão de seu caráter mercantil. Porém, em um estudo de 2010, notou-se que já não era mais possível desconsiderar os impactos socioambientais, e o modelo de gestão tradicional de custo e benefício vem se extenuando. Contudo, uma base de dados consolidada pode ser transformada em um instrumento de gestão e gerar uma forma ativa de enfrentar a escassez e deterioração da água (Tundisi, 2008).

À vista disso, a situação atual não é apenas o reflexo das precariedades do agravamento da ocorrência de água doce, mas também da inconsistência de seu gerenciamento. Um exemplo disso é dado por Oliveira, Barbosa e Neto (2013), no Rio Grande do Norte, a partir de meados dos anos 1990, onde foi realizado programas de captação, armazenamento, tratamento e distribuição da água, com o propósito de garantir o abastecimento das populações e das atividades agrícolas.

Todavia, desde a antiguidade, muitos povos convivem com a carência dos recursos hídricos (Silva et al., 2014), mas na situação brasileira, de acordo com Grafton et al. (2013), é nas últimas décadas que tem havido um declínio acentuado na disponibilidade de água. Diante desses aspectos, instrumentos técnicos na gestão da água vêm recebendo cada vez mais destaque (Rodrigues et al., 2015).

\subsection{Governança da escassez de água}

É certo que, as chuvas abaixo da média estão contribuindo para a intensificação da gravidade da oferta de água, que hoje são caracterizadas como críticas, fato esse também notado por Jacobi, Cibim e Leão (2015). Porém, são muitos os cursos d'água, só na cidade de São Paulo totalizam-se mais de cem afluências dos rios e das bacias que drenam as represas.

Segundo Martins (2015), em um período de quase três décadas, as academias destinadas aos estudos da água, contribuíram de modo decisivo para a expansão das fronteiras da moderna governança da água, na qual pode ser vinculada a manutenção da vegetação florestal, pois elas permitem a melhor retenção de água em bacias hidrográficas, reduzindo a ação erosiva no solo e 
alimentando os mananciais subterrâneos. Sua destruição pode, nas estações chuvosas, estar associada às causas de inundações e aos deslizamentos, e nos períodos de redução pluvial às secas.

Como comprovado por Souza, Silva e Barbosa (2014), a governança da água é construída socialmente pelas percepções e conhecimentos dos indivíduos, no entanto, o que se nota é uma destruição do meio ambiente, a qual vem atingindo, não somente a dimensão ambiental, mas a econômica também. O que, para Fracalanza, Jacob e Eça (2013), é o ponto central da moderna governança, particularmente sobre a valorização da água enquanto elemento natural, não apenas quanto ao seu valor econômico.

A importância dessa nova gestão se deu ao número crescente de conflitos em virtude da disputa pelo recurso, intensa urbanização, escassez, infraestrutura, em muitas áreas urbanas há até $30 \%$ de perdas na rede após o tratamento das águas, mudanças climáticas e ineficiência e ineficácia da gestão da água (Tundisi, 2008; Campos \& Fracalanza, 2010). Além disso, a sociedade sempre dependeu da água doce para a sobrevivência, desenvolvimento cultural e econômico, e durante toda a história teve-se construções de reservatórios, canais e poços, porém devido ao crescimento populacional e a escassez dos recursos hídricos, aliado ao uso irracional e inadequado da água, os sistemas adutores não estão sendo suficientes para superar o problema do abastecimento dos mananciais hídricos.

Porém, se a disponibilidade de água continuar a cair, resultará em mais degradação ambiental, o que foi ponto de partida para as mudanças na governança dos recursos hídricos, que outorga, de forma constitucional, critérios para o uso de água e um sistema nacional do seu gerenciamento, descentralizando as práticas gestoras e participações em distintos setores sociais, nos âmbitos União, Estado e Distrito Federal (Costa \& Mertens, 2015). Além disso, dentro dessa gestão destacam-se os modelos hidrológicos, por exemplo, o Soiland Water Assessment Tool (SWAT), cujas variáveis dependem do tempo e espaço e permitem uma avaliação continuada dos fenômenos hidrológicos envolvidos.

A Agência Nacional de Águas (ANA) destaca que alguns municípios, principalmente da região Nordeste e da Macro metrópole Paulista apresentam maior instabilidade hídrica, (Jacobi, Cibim \& Leão, 2015), devido a estruturação urbana provoca significativa degradação dos recursos hídricos, com a baixa e irregular garantia do abastecimento das áreas de mananciais. Sabendo disso, considera-se que as instituições são baseadas em entendimentos coletivos, através da socialização, processo o qual catalisa a percepção da realidade atual da água no Brasil (Souza, Silva \& Barbosa, 2014), pois é o recurso natural mais afetado dos problemas ambientais. Portanto, há importância em se discutir esses conceitos, muitas vezes associando com temas como, por exemplo, o uso e ocupação do solo, renda, saneamento ambiental e áreas de risco, além disso examinar como a forma de ocupação do espaço urbano vincula a desigualdade de acesso dos recursos naturais.

Logo, a moderna governança da água, passou a interrogar, a maneira como era gerenciado o uso da água; a localização dos centros de abastecimento; o foco das políticas de água; e os atores considerados e incluídos neste processo. Além disso, traz a preocupação de modificar a atual situação hídrica brasileira, pois se não houver uma intervenção, essa 
circunstância contribuirá para, por exemplo, ao aumento das fontes de contaminação, o que por consequência ocasionará um aumento da vulnerabilidade da população; e a diminuição da disponibilidade de água, que dificultará o acesso à água de boa qualidade.

\section{METODOLOGIA}

Esta pesquisa é classificada como uma análise bibliométrica, uma vez que se buscou quantificar a produção científica e sua disseminação, de natureza básica, pois objetiva-se gerar conhecimentos novos, úteis para o avanço da Ciência, sem aplicação prática prevista, além de envolver verdades e interesses universais, possui abordagem quantitativa, pois há preocupação com mensurabilidade, causalidade, generalização e replicação, exploratória, uma vez que visa proporcionar maior familiaridade com o problema, com vistas a torná-lo mais explícito e descritiva, por analisar de forma sucinta relações entre variáveis com o intuito de desvendar a ocorrência de determinado fenômeno em estudo, contendo registros a respeito do que foi observado.

A amostra de artigos foi definida a partir da escolha da ferramenta de busca, da identificação dos descritores de busca, de trabalhos com pelo menos uma citação, da abordagem da governança no Brasil e de apenas artigos científicos e publicados, conforme ilustrado no fluxograma da Figura 1.

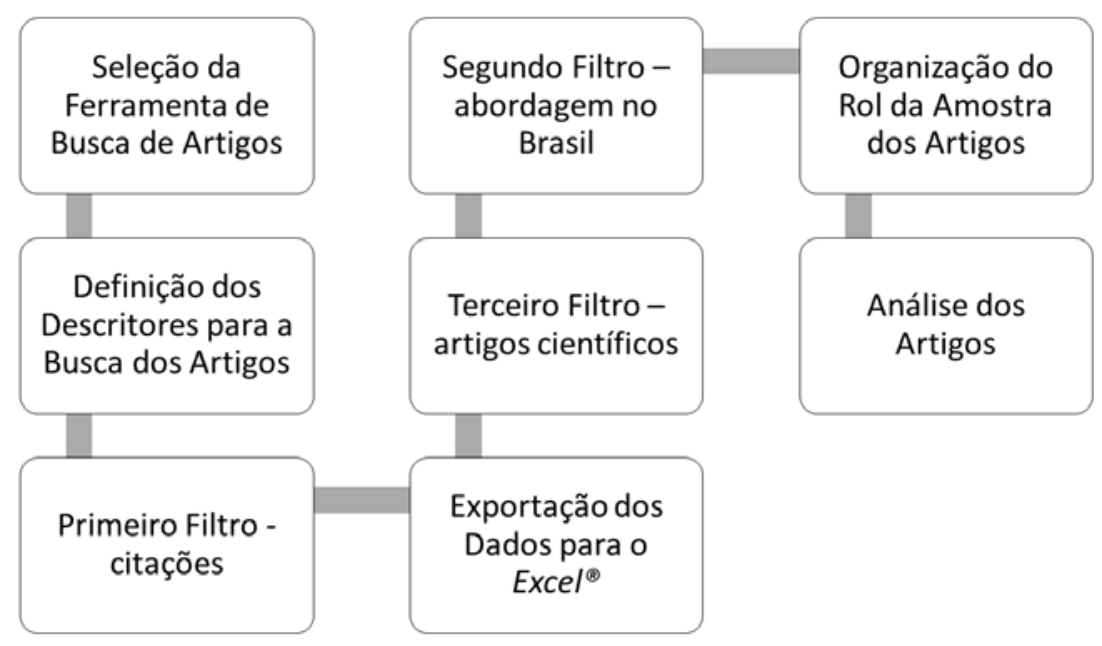

Figura 1: Fluxograma do processo de definição, organização e análise da amostra.

Utilizou-se a ferramenta de pesquisa Google Acadêmico, como principal fonte de informação da pesquisa. A opção pela utilização dessa ferramenta leva em conta critérios de tradição, credibilidade e visibilidade, além disso, essa ferramenta tem como característica a leitura do comportamento cientifico da área. Procedeu-se a busca utilizando-se os descritores governança água OR governança hídrica, foram encontrados 4.430 trabalhos. Fez-se uma outra busca com os termos em inglês water governance brazil, o qual retornou os mesmos resultados.

Quanto ao refinamento, para a primeira etapa, utilizou-se o software Publish or Perish ${ }^{\circledR}$ que retorna os 1000 trabalhos mais relevantes cientificamente e já exclui os duplicados que por ventura podem aparecer na busca. Esse software, também fornece a opção de excluir aqueles que não foram citados, o que resultou em 278 trabalhos. Esse critério foi colocado como 
refinamento da amostra afim de analisar trabalhos ainda mais relevantes, além disso, a probabilidade de trabalhos não citados forem artigos é muito baixa.

Ao que concerne ao tipo de abordagem, filtrou-se para aqueles que apresentavam um contexto nacional, o que retornou 206 e no que se refere à determinação do tipo de publicação optou-se por apenas artigos científicos publicados, resultando em uma amostra final de 137 artigos.

Nessa pesquisa, foram aplicadas algumas leis clássicas da pesquisa bibliométrica, de acordo com Araújo (2006). A lei de Zipf, fundamentada na verificação da frequência que determinadas palavras aparecem em um conjunto de textos, a Lei de Lotka que trata da produtividade cientifica de autores e a Lei de Bradford, que se refere à produtividade de periódicos.

\section{RESULTADOS E DISCUSSÕES}

Uma primeira categoria de análise dos resultados (Figura 2) visa identificar tendências de crescimento ou decréscimo no interesse de desenvolvimento de estudos na área, classificando os artigos da amostra de acordo com o ano de publicação. Observou-se que a primeira publicação relacionada a governança da água data do ano de 1999. Nota-se um pequeno ciclo de crescimento entre os anos 2000 e 2004, interrompido em 2005 e um segundo ciclo mais longo iniciado em 2006 e que se sustentou até 2011, ano com maior volume de publicações. Apesar da tendência de decréscimo no número de publicações verificada nos últimos anos, no período de 1999 a 2015, o crescimento acumulado foi de 700\%. Ressalva-se que o ano de 2016 inclui apenas os resultados do primeiro semestre.

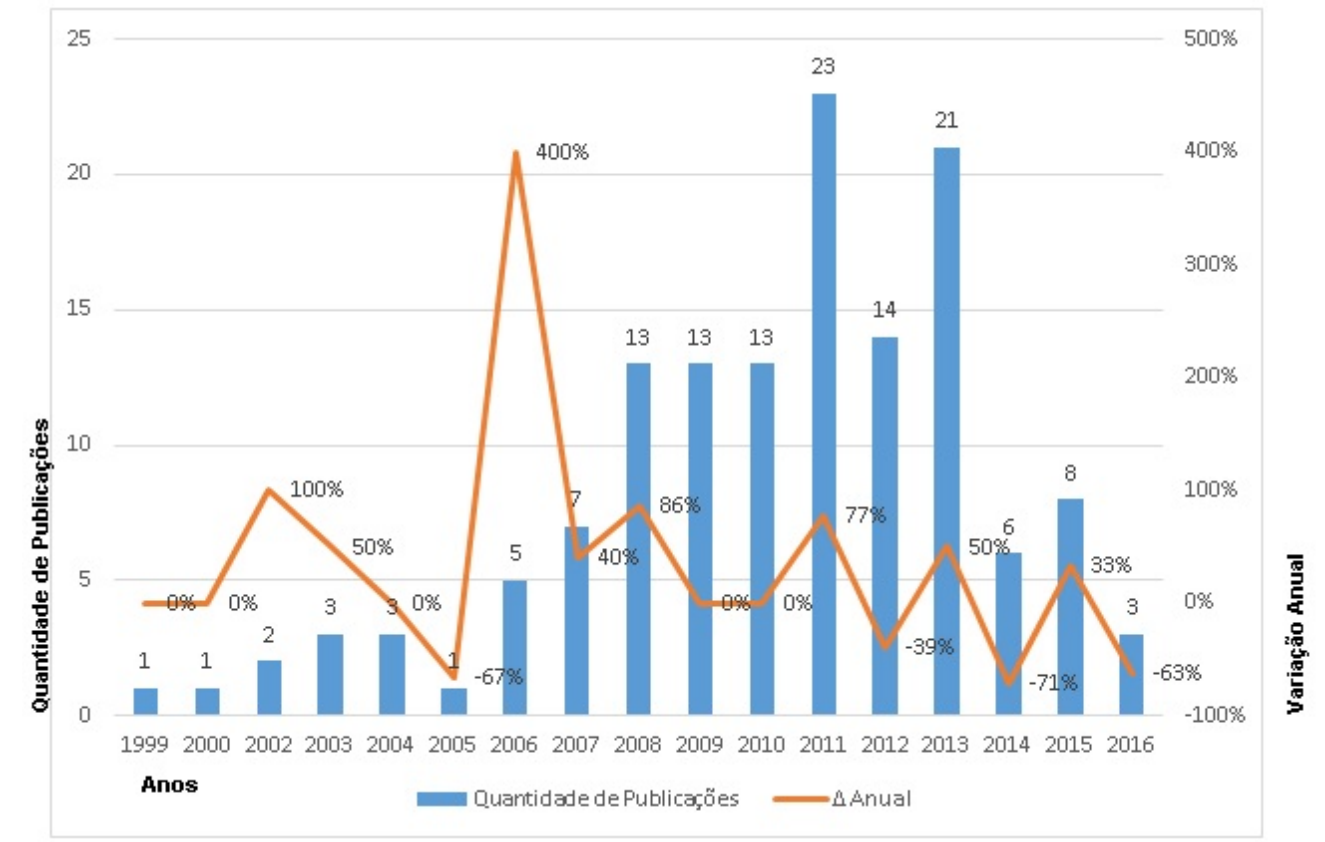

Figura 2. Evolução das publicações da amostra ao longo do tempo (barras azuis) e variação anual em relação ao ano anterior (linha laranja).

Com relação à distribuição dos trabalhos por autor, a análise da amostra revelou uma dispersão considerável. Durante o período analisado, duzentos e cinquenta autores produziram 
pesquisas sobre o tema deste trabalho. A Figura 3 ilustra os autores que foram responsáveis por pelo menos três publicações cada.

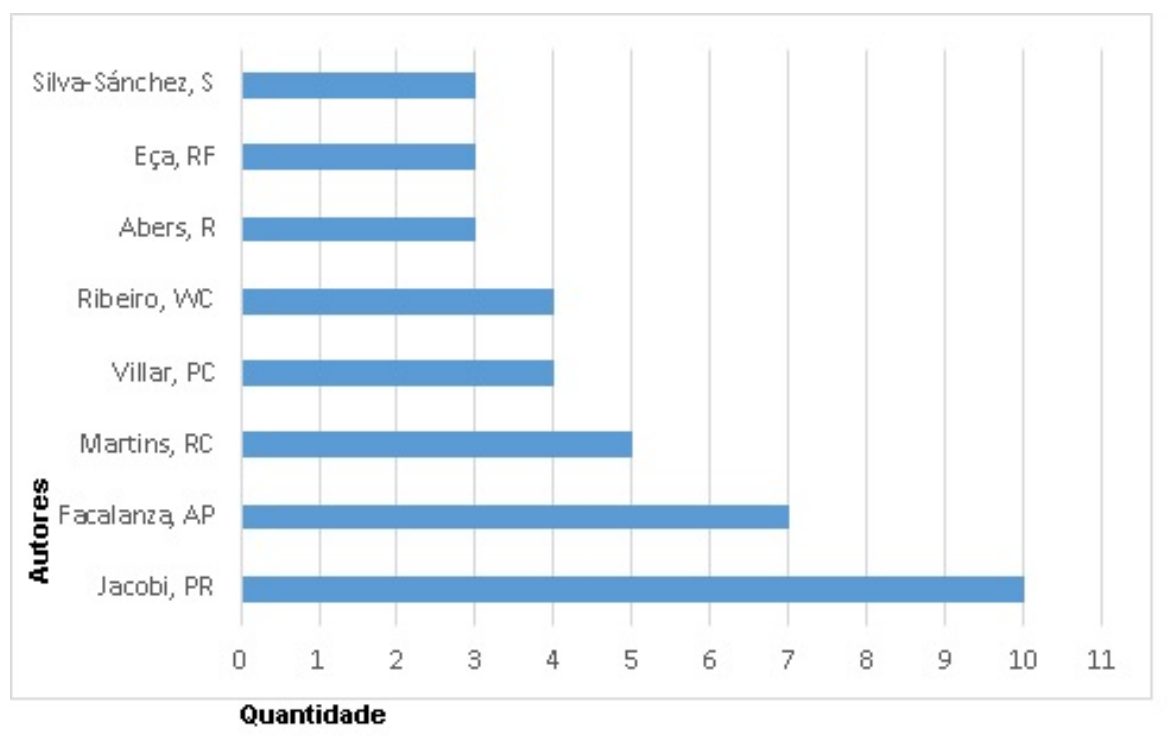

Figura 3. Autores com maior número de publicações da amostra.

A Tabela 2 apresenta a distribuição dos 10 periódicos mais utilizados para a publicação desses 137 artigos da amostra. Com o International Standard Serial Number (ISSN) foi obtido o Fator de Impacto (FI) dos periódicos de acordo com Journal Citation Reports ${ }^{\circledR}\left(\mathrm{JCR}^{\circledR}\right)$ de 2015. Também é apresentada média de citações por artigos (C/A) publicados em cada um dos periódicos. É possível perceber que as médias variam bastante de um periódico para outro e que nem sempre, os periódicos mais utilizados são os mais citados.

Tabela 2: Distribuição dos 10 periódicos mais utilizados pelos pesquisadores da amostra para publicação.

\begin{tabular}{|c|c|c|c|c|c|}
\hline ISSN & Periódico & Citações (C) & Artigos (A) & C/A & FI 2015 \\
\hline $1414-753 x$ & Ambiente \& Sociedade & 199 & 11 & 18,09 & 0,142 \\
\hline 0103-4014 & Estudos avançados & 115 & 8 & 14,38 & 0,227 \\
\hline $0305-750 x / \$$ & World Development & 71 & 1 & 71,00 & 2,100 \\
\hline 2317-1529 & Revista Brasileira de Estudos Urbanos e Regionais & 67 & 5 & 13,40 & - \\
\hline 1982-0259 & Revista Katálysis & 61 & 1 & 61,00 & - \\
\hline $1468-2427$ & International Jour. of Urban and Regional Research & 60 & 2 & 30,00 & 2,267 \\
\hline 0104-1290 & Saúde e Sociedade & 57 & 1 & 57,00 & 0,250 \\
\hline 0016-7185 & Geoforum & 33 & 1 & 33,00 & 1,512 \\
\hline $2236-9996$ & Cadernos Metrópole & 31 & 4 & 7,750 & - \\
\hline 1461-7072 & Development & 29 & 1 & 29,00 & 0,198 \\
\hline
\end{tabular}

Na tabela 3, são apresentados os principais indicadores processados pelo Publish or Perish $^{\circledR}$, com destaque para os índices H e G.

Tabela 3: Principais indicadores da amostra.

\begin{tabular}{ccccccc}
\hline Artigos & Total de Citações & (C)/Ano & (C)/Artigo & Autores/Artigo & Índice H & Índice G \\
137 & 1.120 & 65,88 & 8,18 & 1,82 & 15 & 28 \\
\hline
\end{tabular}

Por meio do índice $\mathrm{H}$ é possível verificar a visibilidade que autores e artigos apresentam a partir das citações que recebem. Essa medida é uma forma de quantificar o desempenho, impacto, visibilidade, qualidade e produtividade dos pesquisadores, de forma confiável e de fácil acesso (EGGHE, 2010). É definido como, o número " $h$ " de artigos de um determinado 
pesquisador tem mesmo número " $\mathrm{h}$ " de citações ou mais. Portando, um índice $\mathrm{h}$ de 15 , indica que a amostra possui 15 artigos com pelo menos 15 citações.

Já o índice G, conforme explica Egghe (2006), atua como complemento ao $\mathrm{H}$ e mede o desempenho de citações de um conjunto de artigos oferecendo assim, mais peso aos artigos altamente citados. Ele representa o quadrado dos artigos mais citados, dessa maneira, o índice $G$ apresentado, significa que houve 28 artigos mais citados na amostra e receberam juntos 784 citações.

\section{CONCLUSÕES}

A transição para a inovação do paradigma de gestão de água requer soluções colaborativas, em que os interesses da coletividade deverão estar acima dos interesses individuais, por exemplo, assegurar o uso sustentável, racional e integrado dos recursos hídricos.

Este estudo apresentou dados de uma busca bibliométrica sobre a governança da água, afim de mostrar a evolução das publicações durante os anos, sendo que o ano de 2011 foi o que teve maior número de publicações, os principais autores, o qual com maior número de artigos foi Jacobi P. R., os principais periódicos, nos quais os trabalhos foram publicados, dentre esses o com mais publicações foi a Revista Ambiente \& Sociedade, que apresenta um fator de impacto de 0,14 . Por fim a representatividade do tema no meio cientifico, o qual destaca-se com 137 artigos, 1120 citações.

A adoção de uma abordagem integrada depende da consciência da sociedade como um todo sobre os benefícios que ela pode obter com a mesma. Além disto, requer um ajuste dos arranjos institucionais, que são estruturados de modo fragmentado. $\mathrm{O}$ que, se, por um lado, a separação institucional e a proposição de políticas setoriais podem ser justificadas por critérios de racionalidade político-administrativa, por outro, implica na descentralização do poder das organizações sociais.

Assim, a governança da água, trata-se da reintegração da natureza na história, pois, a dimensão cultural aponta que são necessárias mudanças nos nossos valores, instituições e modo de vida na medida em que não se pode viver bem se a natureza é destruída.

\section{REFERÊNCIAS}

1. Araújo, C. A. (2006) Bibliometria: evolução histórica e questões atuais. Em Questão, 12(1), 11-32.

2. Campos, V. N. O. \& Fracalanza, A. P. (2010) Governança das Águas no Brasil: conflitos pela apropriação da água e a busca da integração como consenso. Ambiente \& Sociedade, 13(2), 365-382.

3. Costa, A. L. \& Mertens, F. (2015) Governança, redes e capital social no plenário do Conselho Nacional de Recursos Hídricos do Brasil. Ambiente \& Sociedade, 18(3), 153-170.

4. Fracalanza, A. P., Jacob, A. M. \& Eça, R. F. (2013) Justiça Ambiental e Práticas de Governança da Água: (Re) Introduzindo Questões de Igualdade na Agenda. Ambiente \& Sociedade, 16(1), 19-38.

5. Gil, A. C. (2010) Como elaborar projetos de pesquisa. São Paulo: Atlas, 5a Edição. 
6. Grafton, R. Q., Pittock, J., Davis, R., Williams, J., FU, G., Warburton, M., Udall, B., Mckenzie, R., Yu, X., Che, N., Connell, D., Jiang, Q., Kompas, T., Lynch, A., Norris, R., Possingham, H. \& Quiggin, J. (2013) Global insights into water resources, climate change and governance. Nature Climate Change, 3(), 315-321.

7. Jacobi, P. R., Cibim, J., Leão, R. S. (2015) Crise hídrica na macrometrópole paulista e respostas da sociedade civil. Estudos avançados, 29(84), 27-42.

8. Jacobi, P. R., Fracalanza, A. P., Silva-Sánchez, S. (2015) Governança da água e inovação na política de recuperação de recursos hídricos na cidade de São Paulo. Cadernos Metrópole, 17(33), 61-81.

9. Martins, R. C. (2015) Fronteiras entre desigualdade e diferença na Governança das Águas. Ambiente \& Sociedade, 18(1), 221-238.

10. Oliveira, M. A., Barbosa, E. M. \& Neto, J. D. (2013) Gestão de recursos hídricos no Rio Grande do Norte: Uma análise da implementação da política hídrica. HOLOS, 29(1), 3-27.

11. Ottoni, B. M. P., Couceiro, S. R. M., Castro, V. L. L. \& Pereira, R. A. (2011) Outorga do direito de uso dos recursos hídricos no Rio Grande do Norte. HOLOS, 27(1), 57-71.

12. Rodrigues, E. L., Elmiro, M. A. T., Jacobi, C. M. \& Lamounier, W. L. (2015) Aplicação do modelo SWAT na avaliação do consumo de água em áreas de florestas plantadas na bacia do rio Pará, Alto São Francisco, em Minas Gerais. Sociedade \& Natureza, Uberlândia, 27(3), 485500.

13. Silva, J. A. L., Medeiros, M. C. S., Freitas, J. P., Dantas, H. F. S. A. \& Azevedo, P. V. (2014) Gestão hídrica a partir de cisternas de placas: avaliação socioambiental da eficiência do P1MC no município de Pedra Lavrada-PB. HOLOS, 30(4), 47-60.

14. Souza, M., Silva, C. P. \& Barbosa, L. M. (2014) Governança e difusão de normas para a gestão de aquíferos compartilhados: O papel do ISARM. Contexto Internacional, 36(1), 261-289.

15. Tundisi, J. G. (2008) Recursos hídricos no futuro: problemas e soluções. Estudos Avançados, 22(63), 7-16.

16. Wolkmer, M. F. S. \& Pimmel, N. F. (2013) Política Nacional de Recursos Hídricos: governança da água e cidadania ambiental. Sequência, 34(67), 165-198. 